\title{
Breast reconstruction using a laparoscopically harvested pedicled omental flap after endoscopic mastectomy for patients with breast cancer: an observational study of a minimally invasive method
}

\author{
Zi-Han Wang ${ }^{1 \#}$, Pei Xin ${ }^{2 \#}$, Xiang Qu ${ }^{1}$, Zhong-Tao Zhang ${ }^{1}$ \\ ${ }^{1}$ General Surgery Department, Beijing Friendship Hospital, Capital Medical University, Beijing 100000, China; ${ }^{2}$ Surgical Oncology Department, \\ Beijing Chuiyangliu Hospital, Beijing 100000, China \\ Contributions: (I) Conception and design: P Xin; (II) Administrative support: None; (III) Provision of study materials or patients: None; (IV) \\ Collection and assembly of data: ZH Wang; (V) Data analysis and interpretation: ZH Wang; (VI) Manuscript writing: All authors; (VII) Final \\ approval of manuscript: All authors. \\ \#These authors contributed equally to this work. \\ Correspondence to: Xiang Qu, MD. Zhong-Tao Zhang, MD. Capital Medical University, No. 95, Yong'an Road, Xicheng District, Beijing 100000, \\ China. Email: XiangQusa@gmail.com; yyzhongtao@126.com.
}

\begin{abstract}
Background: Breast reconstruction is typically performed using autologous tissue from a laparoscopically harvested omental flap. Because open surgery and another abdominal wall incision for a subcutaneous tunnel cannot be avoided, minimal scars typically cannot be achieved. This study explored a minimally invasive method of pedicled omental flap breast reconstruction in which omentum harvesting, mastectomy, and subcutaneous tunnel establishing were performed laparoscopically and endoscopically, and large incisions on the thoracic and abdominal wall were unnecessary.

Methods: Ten patients with breast cancer were enrolled. They underwent endoscopic subcutaneous mastectomy (ESM) and single-stage breast reconstruction using a laparoscopically harvested pedicled omental flap (LHPOF), which was pulled through a subcutaneous tunnel that was created under laparoscopic vision. The incisions made on the abdominal wall were no wider than $12 \mathrm{~mm}$, and the thoracic wall incisions were no wider than $30 \mathrm{~mm}$. Three of the patients had a prosthetic implant placed for reconstruction at the same time because of the large breast volume, and the omental flaps were used to cover the prostheses.

Results: All patients underwent successful single-stage breast reconstruction surgery, and laparotomy was not required. Eight of the patients $(80 \%)$ had satisfactory aesthetic results (five had excellent results and three had good results). The incisions at the thoracic wall and in the donor site area were short and hidden. The mean operation time was $367.6 \mathrm{~min}$ and the mean time for harvesting the omental flap was $62.9 \mathrm{~min}$, similar to previous studies. The total mean blood loss was $37.0 \mathrm{~mL}$. No serious donor-site complications occurred.

Conclusions: LHPOF breast reconstruction combined with ESM is minimally invasive, and satisfactory aesthetic results are achievable. In patients who undergo ESM combined with prosthetic implant reconstruction, the pedicled omental flap can be used to cover the prosthesis instead of using acellular dermal matrix.
\end{abstract}

Keywords: Endoscopy; mastectomy; laparoscopy; omentum; prosthetic implants; mammoplasty

Submitted Dec 07, 2019. Accepted for publication Mar 27, 2020.

doi: $10.21037 /$ gs.2020.04.06

View this article at: http://dx.doi.org/10.21037/gs.2020.04.06 


\section{Introduction}

Breast reconstruction after mastectomy can be effectively performed using either prosthetic materials or autologous tissue. With the development of reconstructive oncology techniques, the use of autologous tissue has become popular. However, some autologous tissues such as latissimus dorsi flaps, transverse rectus abdominis muscle flaps, and deep inferior epigastric perforator flaps have disadvantages, for example, aesthetic defects, muscle atrophy after irradiation, and functional impairment (1-3). The pedicled omental flap is another choice for patients and surgeons; it has been used for decades and is simple, safe, reliable, and has good contour preservation $(2,4)$. As laparoscopic techniques have been widely used since the beginning of this century, researchers have proven the effectiveness and safety of using a laparoscopically acquired omental flap for breast reconstruction $(5,6)$. Because of the small incisions required, more researchers have started to perform such reconstruction surgery after subcutaneous mastectomy or breast-conserving surgery (BCS), and studies have gradually confirmed the advantages of using a laparoscopically harvested pedicled omental flap (LHPOF) (2,4,7-12). Previous studies have shown that LHPOF is safe, minimally invasive, does not result in implant-related complications, and is resistant to postoperative irradiation $(4,11,13)$. A recent study by Zaha (14), the largest reported series on oncoplastic surgery with an LHPOF, showed the promising oncological safety and clinical benefits of this procedure. The free omental flap used for breast reconstruction in this procedure has been proven to be reliable and safe $(1,15)$, and LHPOF has been shown to cause the least amount of donor site deformity $(5,10,13,14,16,17)$. However, in previous studies, either open surgery was used for mastectomy or the omental flap was taken from another incision in the abdominal wall, resulting in obvious scars on the chest and abdominal wall and less than ideal aesthetic results. Furthermore, most studies in the literature are about BCS, with few on endoscopic subcutaneous mastectomy (ESM).

In our study, we combined ESM with LHPOF for single-stage breast reconstruction. Rather than pulling the flap through another incision created on the abdominal wall, we pulled the omental flap through a subcutaneous tunnel laparoscopically. This less invasive method of breast reconstruction using a pedicled omental flap allowed breast tumor dissection, pedicled omental flap harvesting, and the establishment of a subcutaneous tunnel using only laparoscopic and endoscopic techniques.

\section{Methods}

From September 2016 to October 2017, ten female patients in our department underwent ESM combined with immediate LHPOF breast reconstruction (three patients had breast implants placed simultaneously). The study was approved by the Bioethics Committee of Beijing Friendship Hospital, Capital Medical University (No. 2016-XJS-001; Beijing, China), and performed in accordance with their guidelines and regulations. Informed consent was obtained from all participants prior to participation in the study.

These patients were selected according to the following criteria: had undergone preoperative evaluation of tumors by ultrasound, mammography, or magnetic resonance imaging; distance between the tumor and nipple was $>20 \mathrm{~mm}$ and there was no skin invasion; had a biopsy histopathology that confirmed the tumor type as carcinoma; absence of distant metastasis including metastases to the lung, liver, and bone; no history of upper abdominal surgery; and desire to repair the breast deformity or defect. The gastroepiploic vascular communicating branches were evaluated preoperatively using three-dimensional reconstructed images (Figure 1). Good communication with the branch between the left and right gastroepiploic vessels was essential for survival of the transferred flap, confirming that the right gastroepiploic artery was the dominant blood supply vessel. Indocyanine green angiography (ICGA) was used to observe the blood supply of the omental flap during the operation, and Doppler examination was performed postoperatively to monitor the condition of the omental flap. All patients underwent bowel preparation before surgery in case of gastrointestinal injury.

The patient was placed in the supine position with the ipsilateral arm at $90^{\circ}$ of abduction and with the ipsilateral body elevated. A carbon nanoparticle suspension was injected around the nipple-areola complex to track the sentinel lymph nodes. ESM and sentinel lymph node biopsy were performed. Then liposuction was performed according to a previous study (18) as follows. Liposuction liquid was used to infiltrate the axillary fat pad $(250-500 \mathrm{~mL})$, subcutaneous fat pad $(1,000-1,500 \mathrm{~mL})$, and anterior fat pad of the pectoralis muscle $(1,000-1,500 \mathrm{~mL})$. After $20 \mathrm{~min}$, a $12 \mathrm{~mm}$ incision was created in the anterior axilla at the inframammary level (port A). Then a liposuction tube was inserted to remove the adipose tissue at a pressure of 


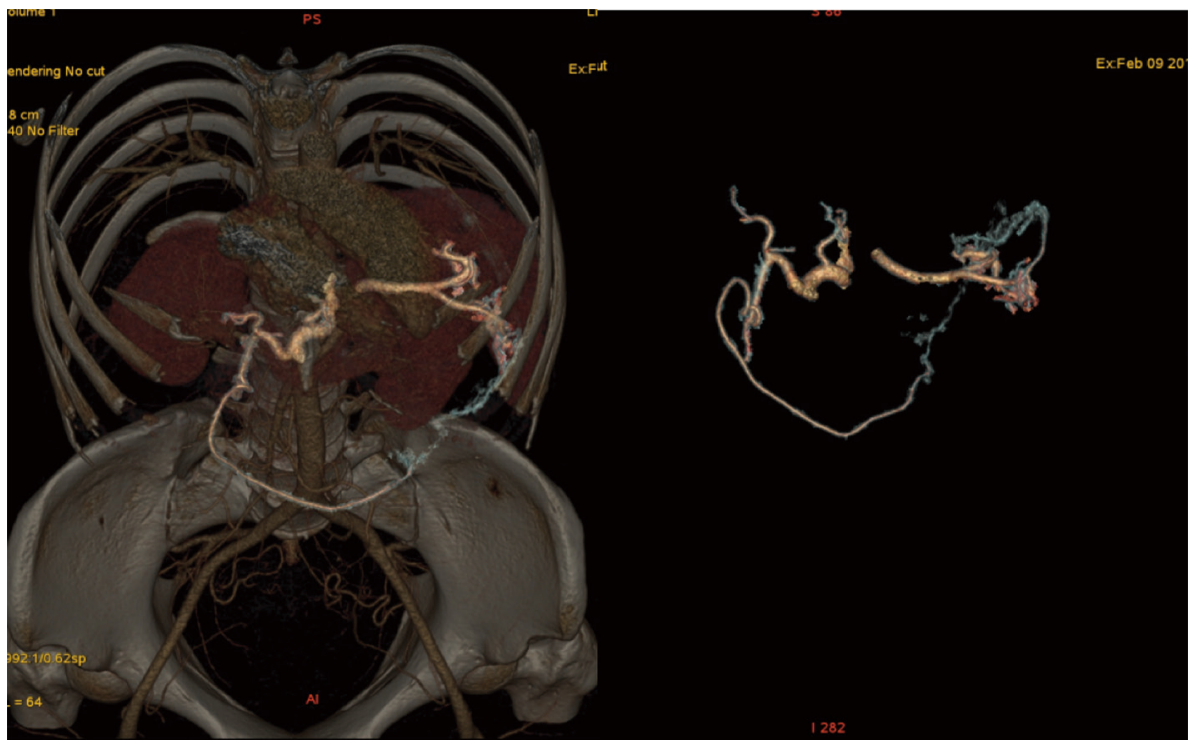

Figure 1 Three-dimensional reconstructed image shows that the right gastroepiploic vessel was the dominant source of blood for the greater omentum. The communicating branches of the gastroepiploic vascular were evaluated.

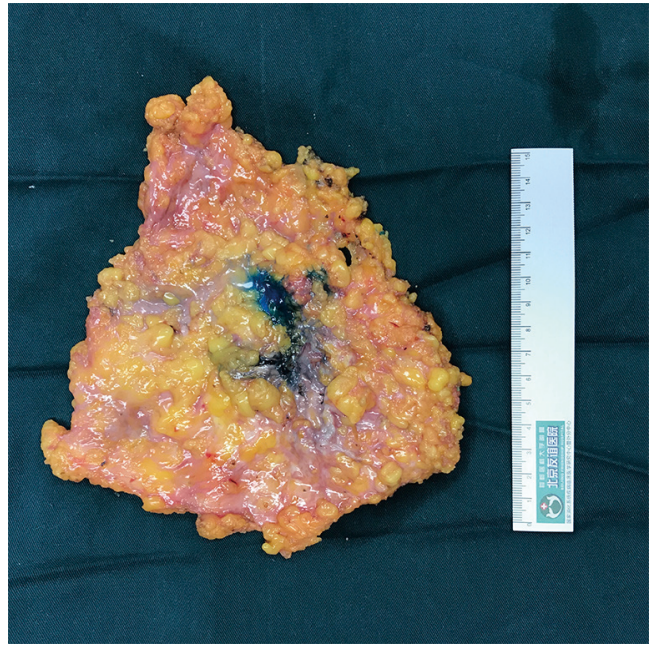

Figure 2 Mammary tissue sample.

800 mbar. Next, a midaxillary incision $(10 \mathrm{~mm}$ at the nipple level; port $\mathrm{C}$ ) and midclavicular incision $[12 \mathrm{~mm}$ at the inframammary fold (IMF); port B] were created, and three trocars were inserted at the three port sites. An adequate working space was created in the breast by insufflation with carbon dioxide gas $(8 \mathrm{mmHg})$ through port $\mathrm{C}$; a $10 \mathrm{~mm}$ optical port was placed at a $30^{\circ}$ angle in port A. Because of the use of liposuction and carbon nanoparticles, blackstained sentinel lymph nodes were easily located and removed through the midaxillary trocar. An endoscopic retrieval device was used to avoid implantation metastasis. Intraoperative frozen section analysis was conducted to determine whether axillary lymph node dissection was required. Mammary gland resection was performed by severing Cooper's ligaments and the fibrous connections with the pectoralis major muscle. Then the mammary gland was removed with a second endoscopic retrieval device from the port $B$ incision, which was extended to $30 \mathrm{~mm}$, and this incision was reused in the following step in which the omental flap was removed (Figure 2). The stump of the nipple site and the dissected surfaces of the tumor (bordering the skin and the pectoralis major muscle) were submitted for frozen section pathological analysis. Outcomes of the frozen section determined whether the dissection range needed to be enlarged.

To create the LHPOF, an umbilicus incision was made and a $12 \mathrm{~mm}$ trocar was inserted to create the camera port. The pneumoperitoneum was established at a pressure of $12 \mathrm{mmHg}$. Laparoscopic inspection was performed to observe the condition of the greater omentum in terms of size, vascular supply, and adhesion to the peritoneum or other organs. After exploration, another two or three 5 -mm incisions were made for the operating ports. The incision at port $\mathrm{B}$ on the chest wall was made for use as a trocar entrance during abdominal surgery if necessary for omental flap harvesting, and the trocar tunnel was used for 

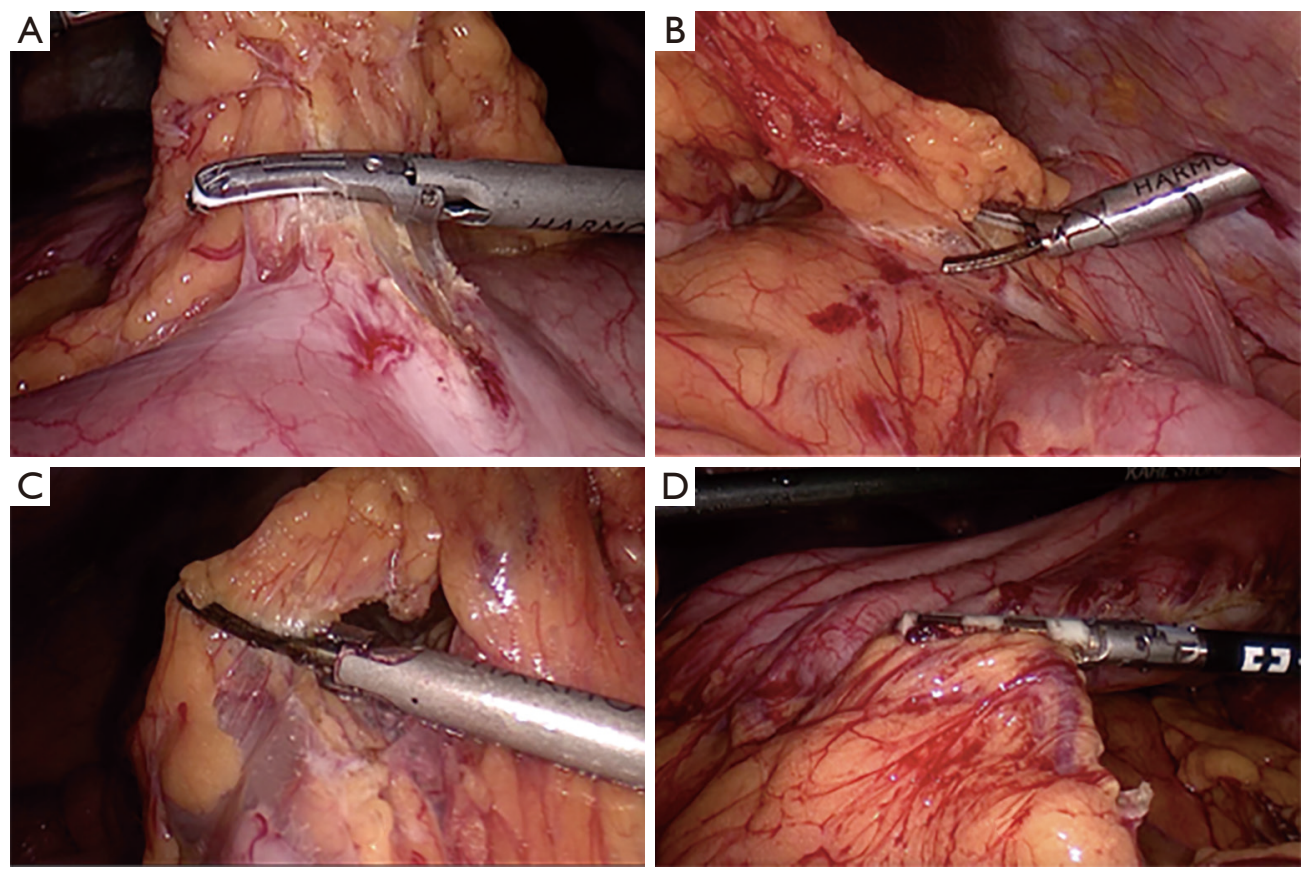

Figure 3 Isolation of the greater omentum. (A) The middle of the transverse mesocolon was the starting point, and then we moved towards the left. (B) The middle and left gastroepiploic vessels were identified and cut. (C) Isolation of the adhesion between the transverse mesocolon and greater omentum. (D) The hemal arch was preserved as much as possible along the omentum.

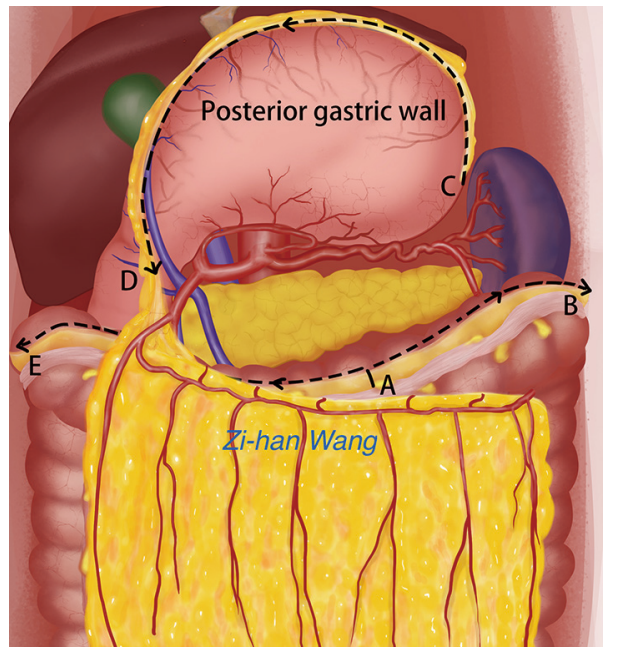

Figure 4 The "line" of the harvesting pedicled omental flap.

establishing a subcutaneous tunnel in the subsequent step. An incision was made $1 \mathrm{~cm}$ superior to the transverse colon wall at the left of middle point. As the space between the anterior lobe of the transverse mesocolon and the omentum was clearer on the left than on the right, the omentum was dissected towards the left (Figure 3). The appearance of the posterior gastric wall indicated that the correct space had been located, thereby reducing the chance of transverse mesentery injury. The left and middle gastroepiploic vessels were blocked and carefully cut to avoid splenic injury. The hemal arch formed by the branches of the gastroepiploic vessels was carefully preserved, and dissection was performed as close as possible to the gastric wall and up to the site of the greater curvature until the pyloric ring was passed. The right gastroepiploic vein and artery were carefully protected as vessel pedicles (Figure 4).

To establish a subcutaneous tunnel, the incision for port B (which was previously enlarged to $30 \mathrm{~mm}$ ) was used as the exit for the omental flap. We separated the peritoneum and the rectus abdominis muscle laparoscopically with a harmonic scalpel. The incision width was determined by the pedicle size, and was usually $25-30 \mathrm{~mm}$. We maintained a space of $5 \mathrm{~mm}$ between the incision and omentum to reduce the incidence of incisional hernia and to protect the pedicle from compression. We laparoscopically expanded the subcutaneous tunnel towards the abdominal cavity from the outer incision (port B incision) to establish a subcutaneous tunnel between the inner and outer incisions (Figure 5); the width of the tunnel was about $25-30 \mathrm{~mm}$. 


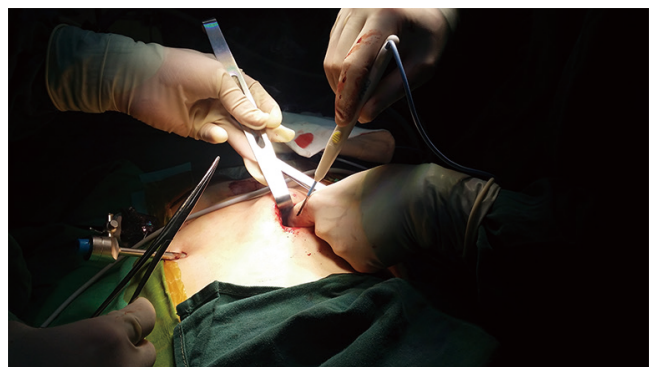

Figure 5 The inner incision was made next to the rectus abdominis muscle. The outside incision was at the level of the inframammary fold. In all cases, we extended the midclavicular incision to $30 \mathrm{~mm}$ to create the outside incision, and an electric scalpel was used to isolate the tunnel by removing the subcutaneous adipose tissue as needed until the tunnel was connected to the inner incision.

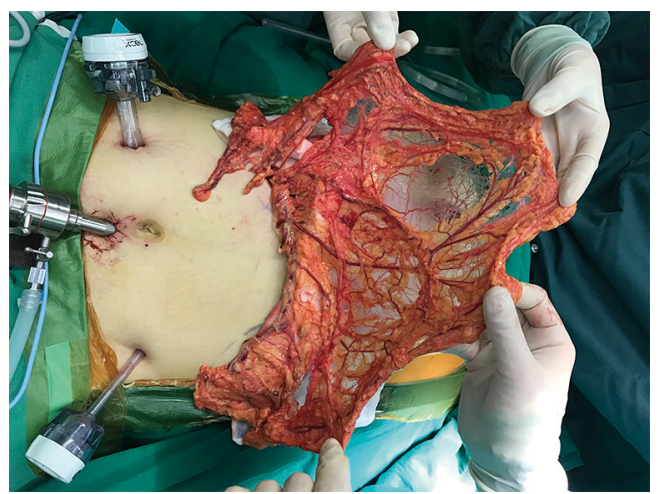

Figure 6 The greater omentum was pulled out through the subcutaneous tunnel. The blood supply and color of the omentum were carefully examined to ensure that there were no signs of necrosis.

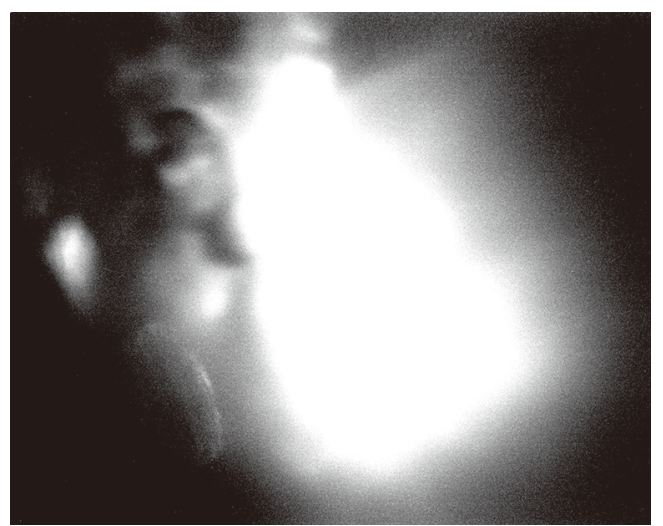

Figure 7 After 3 min of injecting the indocyanine green, the omental flap was enhanced by indocyanine green angiography fluorescence, which showed that there was no blood flow disorder.

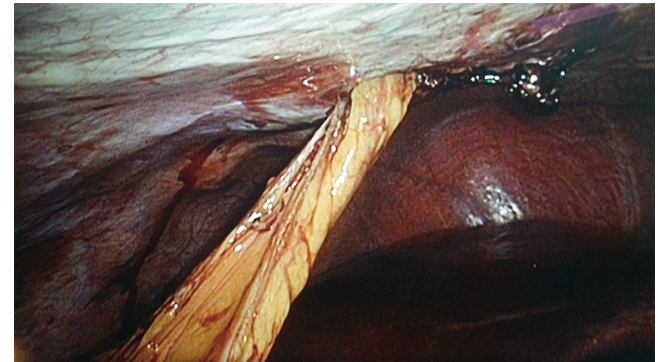

Figure 8 The omentum pedicle was kept in a relaxed state while the pneumoperitoneum was maintained, so that a pulling sensation and bowel dysfunction could be avoided. The omentum pedicle should not be too wide; the appropriate width is $25-30 \mathrm{~mm}$.

Then the prepared greater omentum flap was passed through the tunnel and pulled out using a sponge forceps (Figure 6). Indocyanine green was injected through the peripheral vein to examine the blood supply of the omental flap; the fluorescence intensity represented the blood flow of the total or partial flap (Figure 7). Any weak parts of the fluorescent omental flap were resected. After ensuring that there was no blood flow disorder, the greater omentum was placed into the mastectomy defect. We carefully avoided twisting the omentum pedicle and gastroepiploic vessels, and ensured that there was no tension on the omentum pedicle in the abdominal cavity to avoid dragging pain and intestinal complications (Figure 8).

After ESM, breast reconstruction was performed. If the breast volume was $<150 \mathrm{~mL}$, the omental flap was used as an autogenous tissue flap. In our clinical experience, the volume of the omental flap is commonly $<150 \mathrm{~mL}$; thus, if the breast volume is $>150 \mathrm{~mL}$, the volume of the omental flap is insufficient. In such a case, we would not choose the omental flap alone to reconstruct the breast. Typically, we combine the pedicled omental flap with the prosthetic implant for breast reconstruction. In this study, the omental flap was shaped to adequately fill the breast defect caused by ESM. Then several fixations were performed to attach the omental flap to the thoracic wall. For breast reconstruction combined with prosthetic implant after ESM, the greater omentum was insufficient to rebuild a breast that was symmetrical to the opposite side in patients with a breast volume $>150 \mathrm{~mL}$. Thus, in these patients, a prosthetic implant was placed. Then the pectoralis major muscle was cut off from its inferior margin and stitched to the omental flap. At this point, the omental flap was affixed to the serratus anterior and the IMF, and the implant was covered by the omental flap and the pectoralis major 
muscle, creating a pocket to hold the implant instead of using acellular dermal matrix (ADM). A closed suction drain was placed over the omental flap. If axillary lymph node dissection was performed, then another drain was placed in the axillary area. Both drainage tubes were inserted through the trocar site, and the other endoscopic incisions in the thoracic and abdominal walls were closed.

Photographs were taken before and after surgery from three different angles every 3 months. Patient satisfaction with the aesthetic results was assessed 3 months after surgery using the following five-item, four-step scoring system to evaluate the cosmetic outcomes (19): asymmetry, breast shape, nipple shape, condition of the skin, and wound scar. Each item was evaluated using the following scale: poor, 0; fair, 1; good, 2; and excellent, 3. Scores of each of the five items were totaled for each patient, and the total scores were defined on the basis of a maximum score of 15 (excellent, 15; good, 11-14; fair, 6-10; and poor, 0-5).

\section{Results}

All ten patients underwent successful laparoscopic harvesting of the pedicled omental flap, ESM, and subsequent one-stage breast reconstruction. There was no conversion to laparotomy, and no other incisions were required in the abdominal wall or thoracic wall to establish a subcutaneous tunnel for the omental flap. Three patients underwent breast reconstruction with a prosthetic implant. The mean operation time was $357.7 \mathrm{~min}$ (range: 150.0 to $495.0 \mathrm{~min}$ ). Two patients had sentinel lymph node metastasis and underwent subsequent axillary lymph node dissection. The mean time for harvesting the omental flap was $62.9 \mathrm{~min}$ (range: 32.0 to $95.0 \mathrm{~min}$ ). The total mean blood loss was $37.0 \mathrm{~mL}$. According to ICGA and Doppler sonographic examination, all flaps had good blood supply and survived well without any partial loss. Patients were allowed to eat and walk the day after surgery, and their bowel function recovered 3 days after laparoscopic harvesting of the pedicled omental flap. The mean drainage volume of the resected breast cavity was $192.0 \mathrm{~mL}$ (minimum: $56.0 \mathrm{~mL}$ ), and the drainage tube was withdrawn after a mean of 8.5 days (Table 1 ).

All patients had three or four negligible donor site scars on the abdominal wall, which were similar to those resulting from laparoscopic cholecystectomy. The incisions were also short and hidden and the aesthetic effect was remarkable in the breast reconstruction area. Patient satisfaction at 3 months after surgery is summarized in Table 2 .
All ten patients were satisfied with their postoperative negligible incision scars. Of the eight patients who reported satisfactory reconstruction outcomes (Figures 9,10), five indicated excellent results and three indicated good results. One patient had a fair outcome because of insufficient greater omentum volume, resulting in unsatisfactory symmetry and breast shape. This patient had a lower body mass index; therefore, the results were acceptable and secondary surgery to perform refilling was not necessary. One patient had poor breast reconstruction results because the omental flap partially slid down the breast envelope to the IMF area; therefore, the reconstructed breast and contralateral breast were not symmetrical. Secondary surgery was performed 3 months later to reshape the omental flap.

The follow-up period ranged from 14.5 to 27.7 months (mean: 22.0 months), during which time no omental flaps or fat necrosis occurred. In the donor site area, no pulling sensation or bowel dysfunction (bowel obstruction, postprandial epigastric discomfort) occurred. Two patients had bulging under the subcutaneous tunnel because of thick adipose tissue and a broad omental flap pedicle; therefore, we removed the appropriate subcutaneous adipose tissue. One patient experienced movement of the omental pedicle in the subcutaneous tunnel when coughing, because the inner incision in the abdominal cavity was too large; 1 year after reconstruction surgery, the patient had incisional hernia at the subcutaneous tunnel area and underwent laparoscopic hernia repair. The other patient had abdominal subcutaneous emphysema in the thoracic wall, which was considered a pneumoperitoneum complication; however, it disappeared within 3 months. At the final follow-up examination, neither local recurrence nor distant metastasis was detected in any of the patients. Postoperative conditions of all patients are listed in Table 3 .

\section{Discussion}

LHPOF has been performed for nearly 20 years, and is proven to be safe and reliable, and cause the least amount of donor site deformity compared to traditional methods. LHPOF involves several major procedures including mastectomy, harvesting of the omental flap, establishing a subcutaneous tunnel, and fixation of the pedicled omental flap to the chest wall. However, there are few studies on breast surgery procedures performed by endoscopy and laparoscopy as an alternative to open surgery, especially for establishing a subcutaneous tunnel. In addition, most 


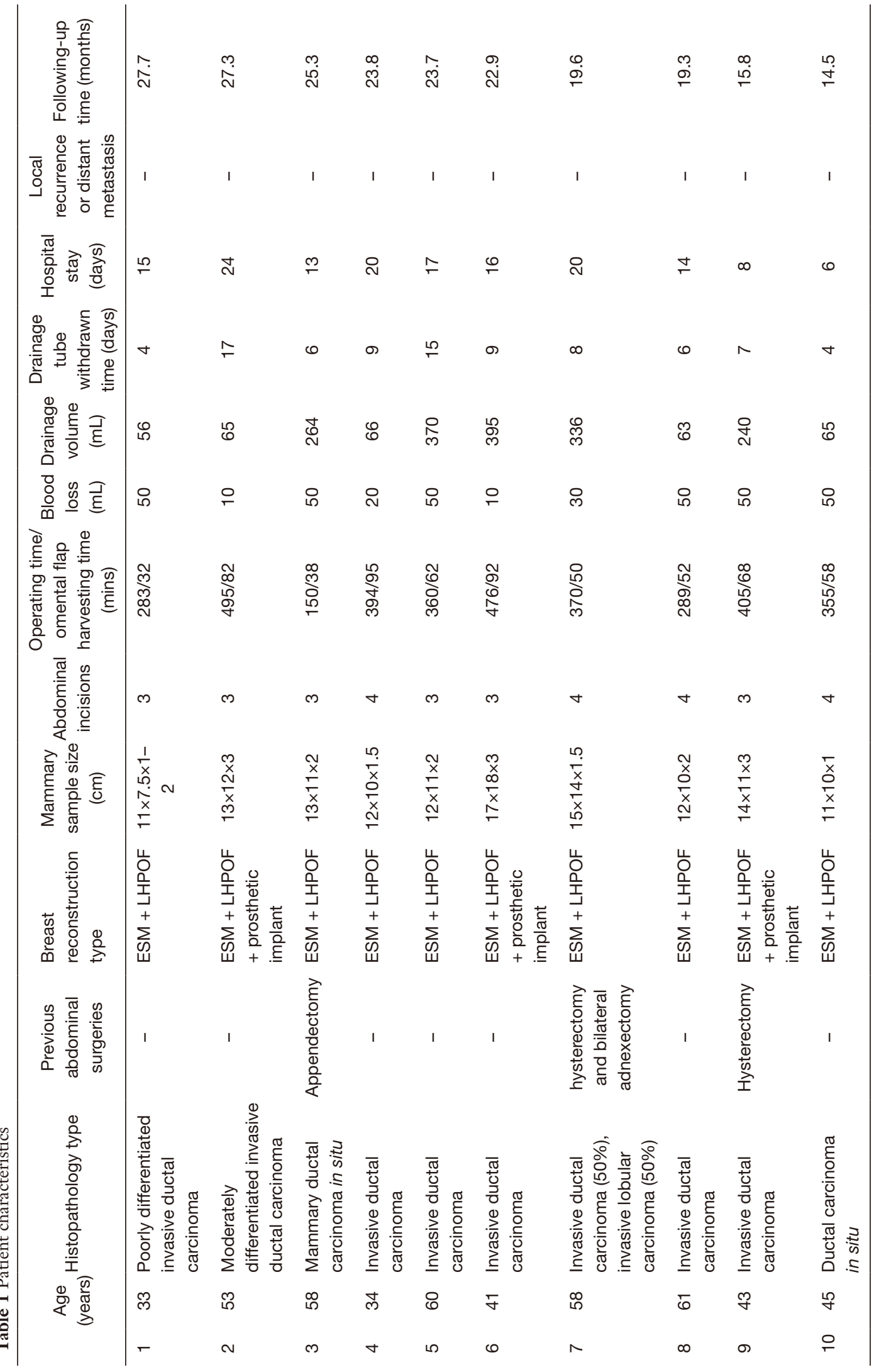


Table 2 Scores of each patient and aesthetic evaluation results

\begin{tabular}{lccccccc}
\hline Patient number & Asymmetry & Breast shape & Nipple shape & Condition of the skin & Wound scar & Total score & Satisfaction \\
\hline 1 & 1 & 1 & 2 & 2 & 3 & 9 & Fair \\
2 & 3 & 3 & 3 & 3 & 3 & 15 & Excellent \\
3 & 2 & 2 & 3 & 2 & 2 & 11 & Good \\
4 & 3 & 3 & 3 & 3 & 3 & 15 & Excellent \\
5 & 2 & 3 & - & 3 & 3 & 11 & Good \\
6 & 3 & 3 & 3 & 3 & 3 & 15 & Excellent \\
7 & 0 & 0 & 0 & 2 & 2 & 4 & Poor \\
8 & 2 & 3 & 3 & 3 & 3 & 14 & Good \\
9 & 3 & 3 & 3 & 3 & 3 & 15 & Excellent \\
10 & 3 & 3 & 3 & 3 & 3 & 15 & Excellent \\
\hline
\end{tabular}
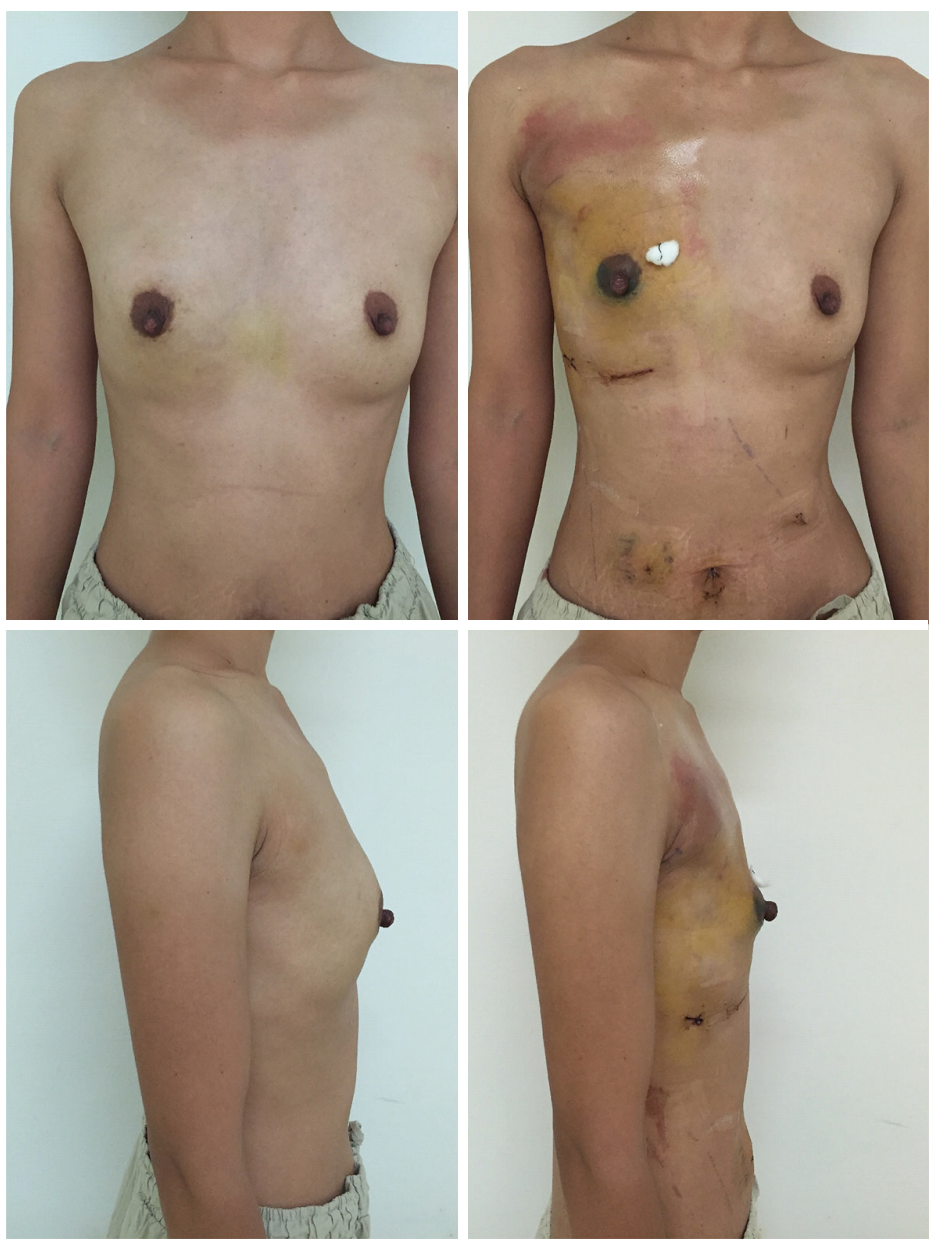

Figure 9 A patient with breast cancer before and after surgery. Endoscopic subcutaneous mastectomy and axillary lymph node dissection were performed, and breast reconstruction was completed using a pedicled omental flap. The greater omentum volume was sufficient to fill the postoperative breast cavity. The patient rated the aesthetic results as good. 


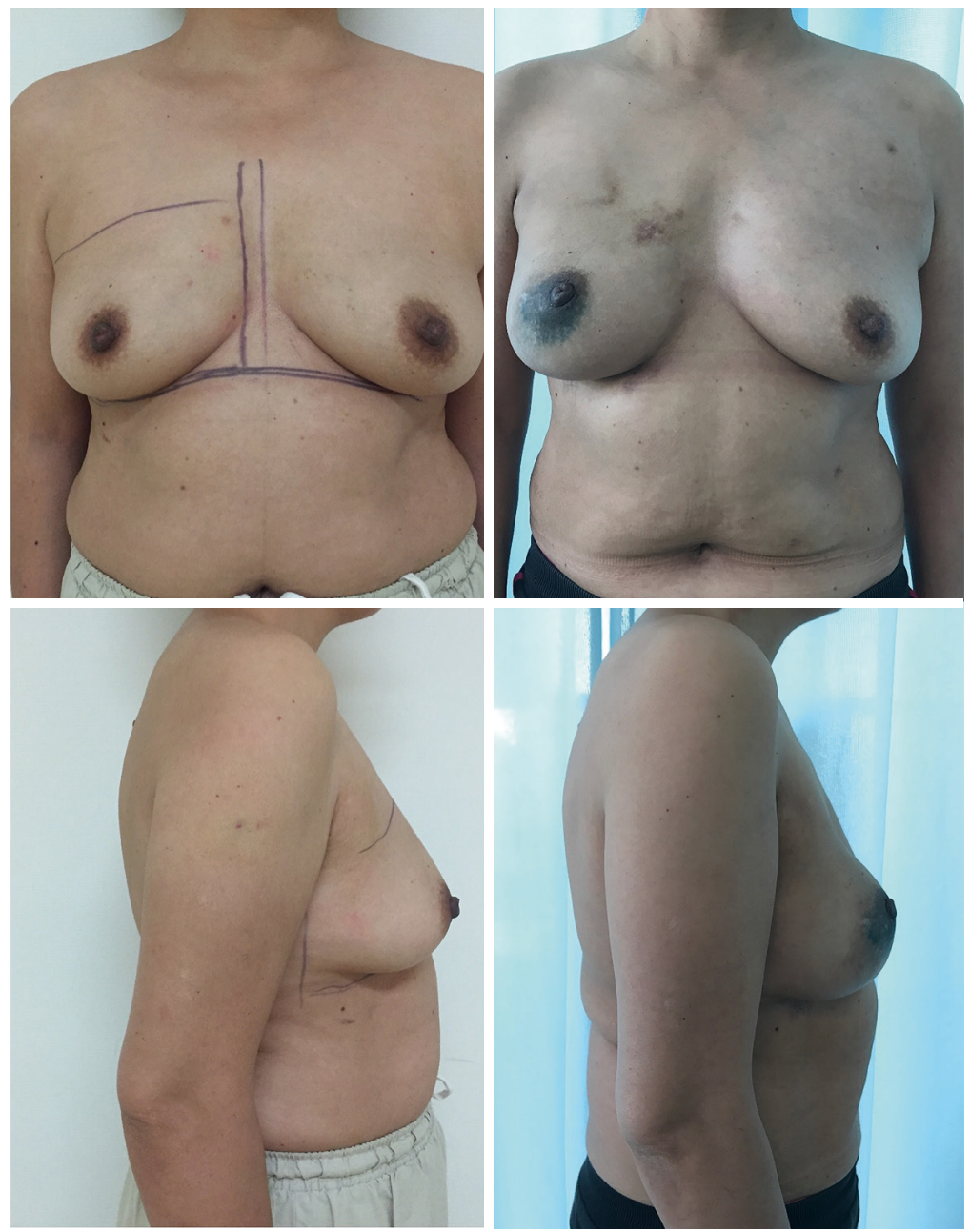

Figure 10 A patient who underwent endoscopic subcutaneous mastectomy and one-stage breast reconstruction, followed by combined pedicled omental flap reconstruction and prosthetic implant placement. Photos were taken before and 3 months after surgery. No scars remained on the thoracic or abdominal wall. The patient rated her satisfaction as excellent.

studies in the literature are about BCS. Our study differs from other studies in two important ways. (I) We tried to perform all procedures (mastectomy, harvesting of the omental flap, establishing a tunnel to transfer the omental flap to the breast) laparoscopically, which has not been done in other studies. No obvious and long incisions were made on the thoracic and abdominal wall; the longest incision was no longer than $30 \mathrm{~mm}$. (II) For patients who had insufficient omentum volume, we combined the pedicled omental flap with prosthetic implant for breast reconstruction. Since the greater omentum has good absorption abilities, this combination surgery can relieve postoperative inflammatory reactions in the operative area, decrease drainage volume, and reduce healthcare expenses.
The greater omentum has many physiological characteristics that make it suitable for surgical reconstruction such as a rich blood supply, softness, a considerable capacity for absorption, resistance to infection, and easy adherence to other tissues. Therefore, it is widely used to cover organ wounds during abdominal surgery and when repairing complex vesicovaginal fistulas (20), as it promotes the healing of wounds created by different surgical approaches. Due to the developments in laparoscopic surgery and microsurgery, the greater omentum has been used for other types of reconstructive surgeries as well. Its first use for breast reconstruction and thoracic wall necrosis after radiotherapy was reported in 1963 (21). However, because a laparotomy with a large left incision is needed, it is not 
Table 3 Postoperative complications

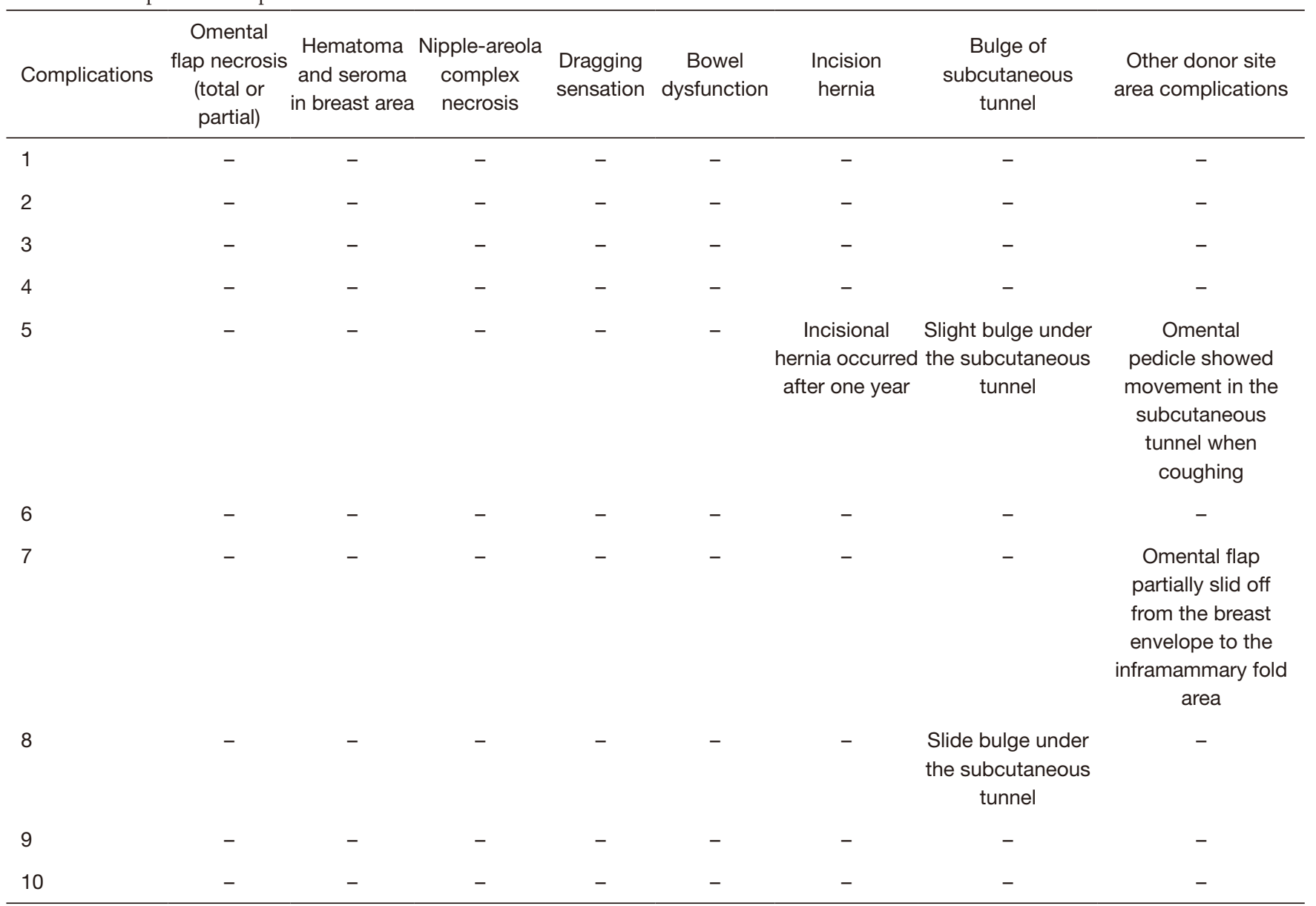

widely used. In 1993, Saltz (22) first reported harvesting the greater omentum laparoscopically to cover a large soft tissue defect in the thoracic wall. Consequently, since the beginning of the $21^{\text {st }}$ century, more breast reconstructions have been performed using a laparoscopically harvested omental flap, and the safety and effectiveness of this procedure have been proven $(3,5,6,10)$.

Although the omentum can be harvested laparoscopically, another vertical incision on the abdominal wall is still typically required to pull out the omental flap. Moreover, the breast tumor is usually resected by open surgery, which requires long incisions on the chest wall and results in unsatisfactory aesthetic results. In our study, a laparoscope and endoscope were used for both parts of the surgery. This method was less invasive and did not require large incisions. The midclavicular incision was reused as the exit point for the omental flap; therefore, an incision in the upper abdominal wall was not needed, and the patients experienced better aesthetic results. Our use of three small incisions instead of open surgery for mastectomy enabled immediate breast reconstruction, better matching with the contralateral breast, and better aesthetic results (23).

We evaluated the gastroepiploic vessel branches, blood supply, and survival condition using a three-dimensional reconstructed technique, ICGA, and postoperative Doppler examination, respectively. Evaluation of the gastroepiploic vessel is essential before surgery to confirm the viability of the right gastroepiploic artery and to reserve it as the vascular pedicle. All ten patients in our study had normal right gastroepiploic arteries, and no anatomical variation of the gastroepiploic vessel was found. During the operation, indocyanine green-enhanced fluorescence showed that there was good blood flow to the total and partial flaps; if a blood flow disorder had been detected at some part of the omentum, this part would have been removed. All patients had good blood supply to the omental flap, and there 


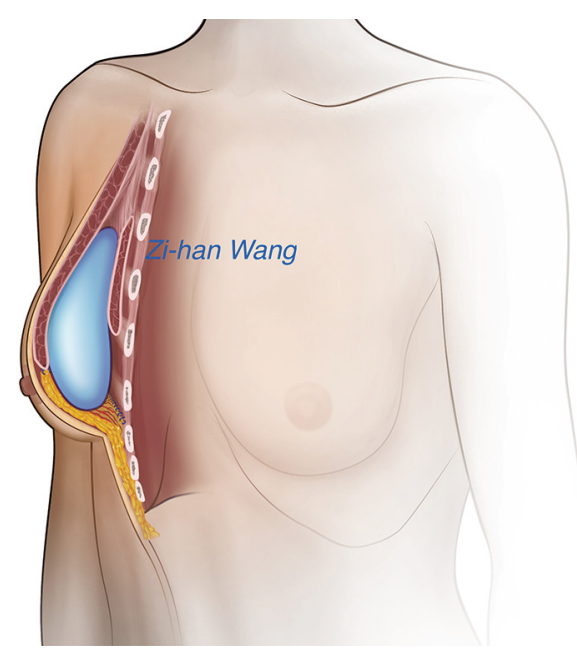

Figure 11 An "intact pocket" was formed by the omental flap, pectoralis major muscle, and lateral serratus anterior fascia.

was no total or partial flap loss. The day after surgery, all patients underwent Doppler examination to confirm good blood flow to the omental flap in the breast envelope. If it had shown a weak blood supply in any part of the flap, the color of the local skin and the characteristics of the drainage fluid would have been evaluated. If the local skin had turned red and the drainage fluid had become cloudy, omental flap necrosis would have been suspected.

In a retrospective clinical study, Kim (2) demonstrated that mini-breast reconstruction using only a pedicled omental flap was successful. In patients with small breast volumes $(<150 \mathrm{~mL})$, the greater omentum is usually sufficient to reconstruct the entire resected breast cavity. In addition, this method is more economical than ADM and lacks serious adverse effects such as implant-related complications. By contrast, in patients with large breast volumes $(>150 \mathrm{~mL})$, the greater omentum is usually insufficient to reconstruct the resected cavity after ESM; therefore, prosthetic breast implants are required. In these cases, the greater omentum serves as a covering layer to wrap the implant; thus, the need for an $\mathrm{ADM}$ or a titaniumcoated polypropylene mesh (TiLOOP) Bra is avoided. The use of ADM is associated with more adverse outcomes requiring surgical intervention (24) such as open evacuation of a hematoma. In this study, after the greater omentum was stitched to the pectoralis major muscle and the lateral serratus anterior, an intact pocket was formed that well encapsulated the implant (Figure 11). The greater omentum showed many advantages in this study: it enhanced the irradiated skin and protected the implant; was more absorbent and decreased the rate of hematoma and seroma information; enclosed the implant and was located behind the nipple-areola complex; and because of its soft tissue pad and good blood supply, the necrosis rate of the nippleareola complex was also decreased. None of the patients in our study had a blood flow disorder of the nipple-areola complex including those with implant reconstruction. Additionally, the breast shape was more natural because it was covered with a thick autologous tissue. The greater omentum also eliminates capsular contracture, of which a $1.2 \%$ incidence rate has been reported (25). In addition to these advantages, breast reconstruction with the greater omentum is more cost-effective, as ADM and the TiLOOP bra are expensive and not always covered by medical insurance in China.

As mentioned above, the greater omentum has good absorption abilities, and thus can be effectively used in the resected breast cavity. An omental flap relieves postoperative inflammatory reactions in the operative area and reduces effusion from the resected breast cavity. The resulting decreased drainage volume and earlier removal of the drainage tube may lead to earlier discharge from the hospital, and accordingly, a reduction in healthcare expenses. In our study, no patient had accumulation of subcutaneous liquid, thereby highlighting one advantage of using the greater omentum for reconstruction.

There were some limitations to this study. First, our follow-up time was short. More time is required to observe the effectiveness and complications of this surgery. Second, this was a retrospective study without a comparison group. In the future, we plan to conduct comparative studies to observe differences between the greater omentum and $\mathrm{ADM}$ or the TiLOOP bra for covering prosthetic implants. Third, we studied a small number of elective cases. More cases are needed to eliminate selection bias and confounding factors. Breast reconstruction after ESM with a LHPOF is a new method available to patients and surgeons that can be combined with prosthetic implant placement if necessary, and is also suitable for cosmetic surgery. This procedure is safe and minimally invasive, with little impairment to the donor site area, and allows patients to achieve satisfactory aesthetic outcomes.

\section{Acknowledgments}

Funding: None. 


\section{Footnote}

Conflicts of Interest: All authors have completed the ICMJE uniform disclosure form (available at http://dx.doi. org/10.21037/gs.2020.04.06). The authors have no conflicts of interest to declare.

Ethical Statement: The authors are accountable for all aspects of the work in ensuring that questions related to the accuracy or integrity of any part of the work are appropriately investigated and resolved. The study was approved by the Bioethics Committee of Beijing Friendship Hospital, Capital Medical University (No. 2016-XJS-001; Beijing, China), and performed in accordance with their guidelines and regulations. Informed consent was obtained from all participants prior to participation in the study.

Open Access Statement: This is an Open Access article distributed in accordance with the Creative Commons Attribution-NonCommercial-NoDerivs 4.0 International License (CC BY-NC-ND 4.0), which permits the noncommercial replication and distribution of the article with the strict proviso that no changes or edits are made and the original work is properly cited (including links to both the formal publication through the relevant DOI and the license). See: https://creativecommons.org/licenses/by-nc-nd/4.0/.

\section{References}

1. Zaha H, Onomura M, Nomura H, et al. Free omental flap for partial breast reconstruction after breast-conserving surgery. Plast Reconstr Surg 2012;129:583-7.

2. Kim H, Yoon CS, Lee HB, et al. Mini-breast reconstruction with an omental flap: a retrospective clinical study. ANZ J Surg 2017;87:E261-5.

3. Romanini MV, Vidal C, Godoy J, et al. Laparoscopically harvested omental flap for breast reconstruction in Poland syndrome. J Plast Reconstr Aesthet Surg 2013;66:e303-9.

4. Khater A. Evaluation of pedicled omental flap delivered through a minilaparotomy for immediate breast reconstruction in obese patients. Aesthetic Plast Surg 2013;37:1140-5.

5. Cothier-Savey I, Tamtawi B, Dohnt F, et al. Immediate breast reconstruction using laparoscopically harvested omental flap. Plast Reconstr Surg 2001;107:1156-63.

6. Jimenez AG, St Germain P, Sirois M, et al. Free omental flap for skin-sparing breast reconstruction harvested laparoscopically. Plast Reconstr Surg 2002;110:545-51.

7. Zaha H, Sunagawa H, Kawakami K, et al. Partial breast reconstruction for an inferomedial breast carcinoma using an omental flap. World J Surg 2010;34:1782-7.

8. Zaha H. Oncoplastic volume replacement technique for the upper inner quadrant using the omental flap. Gland Surg 2015;4:263-9.

9. Zaha, H. Partial breast reconstruction for the medial quadrants using the omental flap. Ann Surg Oncol 2014;21:3358.

10. Zaha H, Inamine S. Laparoscopically harvested omental flap: results for 96 patients. Surg Endosc 2010;24:103-7.

11. Christensen BO, Overgaard J, Kettner LO, et al. Longterm evaluation of postmastectomy breast reconstruction. Acta Oncol 2011;50:1053-61.

12. Zhang P, Luo Y, Deng J, et al. Endoscopic axillary lymphadenectomy combined with laparoscopically harvested pedicled omentum for immediate breast reconstruction. Surg Endosc 2015;29:1376-83.

13. Guan D, Lin H, Lv Z, et al. The oncoplastic breast surgery with pedicled omental flap harvested by laparoscopy: initial experiences from China. World J Surg Oncol 2015;13:95.

14. Zaha H, Abe N, Sagawa N, et al. Oncoplastic surgery with omental flap reconstruction: a study of 200 cases. Breast Cancer Res Treat 2017;162:267-74.

15. Ni C, Zhu Z, Xin Y, et al. Oncoplastic breast reconstruction with omental flap: A retrospective study and systematic review. J Cancer 2018;9:1782-90.

16. Zaha H, Inamine $S$, Naito $T$, et al. Laparoscopically harvested omental flap for immediate breast reconstruction. Am J Surg 2006;192:556-8.

17. Shash H, Al-Halabi B, Aldekhayel S, et al. Laparoscopic Harvesting of Omental Flaps for Breast Reconstruction-A Review of the Literature and Outcome Analysis. Plast Surg (Oakv) 2018;26:126-33.

18. Wang ZH, Qu X, Teng CS, et al. Preliminary Results for Treatment of Early Stage Breast Cancer With Endoscopic Subcutaneous Mastectomy Combined With Endoscopic Sentinel Lymph Node Biopsy in China. J Surg Oncol 2016;113:616-20.

19. Yamashita K. Cosmetic assessment. J Jpn Soc Endosc Surg 2005;10:165-70.

20. Haferkamp A, Wagener N, Buse S, et al. Vesicovaginal fistulas. Urologe A 2005;44:270-6.

21. Kiricuta I. L'emploi du épiploon dans la chirurgie du sein cancéreux. Presse Med 1963;71:15-7.

22. Saltz R, Stowers R, Smith M, et al. Laparoscopically harvested omental free flap to cover a large soft tissue defect. Ann Surg 1993;217:542-6.

23. Sisco M, Kyrillos AM, Lapin BR, et al. Trends and 
variation in the use of nipple-sparing mastectomy for breast cancer in the United States. Breast Cancer Res Treat 2016;160:111-20.

24. Lohmander F, Lagergren J, Roy PG, et al. Implant Based Breast Reconstruction With Acellular Dermal Matrix: Safety Data From an Open-label, Multicenter,
Randomized, Controlled Trial in the Setting of Breast Cancer Treatment. Ann Surg 2019;269:836-41.

25. Salibian AA, Frey JD, Choi M, et al. Subcutaneous implant-based breast reconstruction with acellular dermal matrix/mesh: A systematic review. Plast Reconstr Surg Glob Open 2016;4:e1139.
Cite this article as: Wang ZH, Xin P, Qu X, Zhang ZT. Breast reconstruction using a laparoscopically harvested pedicled omental flap after endoscopic mastectomy for patients with breast cancer: an observational study of a minimally invasive method. Gland Surg 2020;9(3):676-688. doi: 10.21037/ gs.2020.04.06 